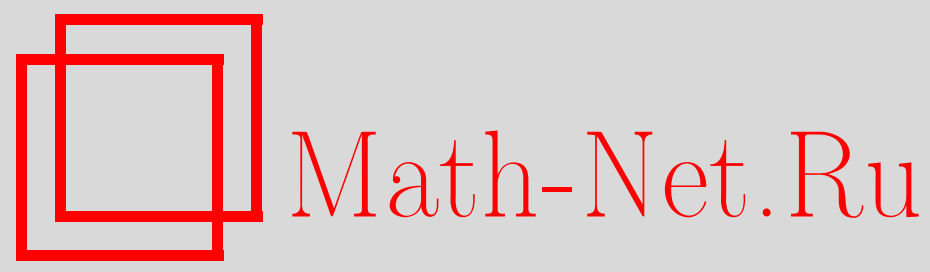

Н. В. Денисова, Полиномиальные по скорости интегралы динамических систем с двумя степенями свободы и торическим конфигурационным пространством, Матем. заметки, 1998, том 64, выпуск 1, 37-44

DOI: https://doi.org/10.4213/mzm1370

Использование Общероссийского математического портала Math-Net.Ru подразумевает, что вы прочитали и согласны с пользовательским соглашением http://www . mathnet.ru/rus/agreement

Параметры загрузки:

IP : 54.205 .225 .156

26 апреля 2023 г., 02:33:47

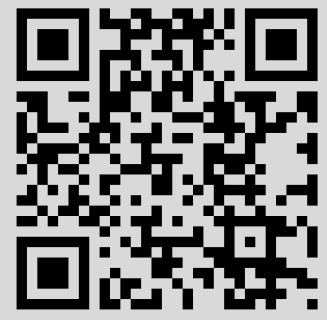




\title{
ПОЛИНОМИАЛЬНЫЕ ПО СКОРОСТИ ИНТЕГРАЛЫ ДИНАМИЧЕСКИХ СИСТЕМ С ДВУМЯ СТЕПЕНЯМИ СВОБОДЫ И ТОРИЧЕСКИМ КОНФИГУРАЦИОННЫМ ПРОСТРАНСТВОМ
}

\author{
Н. В. Денисова
}

В работе рассматриваются динамические системы с двумя степенями свободы с торическим конфигурационным пространством, допускающие полиномиальные первые интегралы по скорости. В работе получены конструктивные критерии существования условных линейных и квадратичных интегралов на двумерном торе. Кроме того, показано, что при некоторых дополнительных условиях степень "неприводимого" интеграла геодезического потока на торе не превосходит 2.

Библиографоял: 9 названий.

1. Введение. В работе рассматриваются динамические системы с двумя степенями свободы, конфигурационным пространством которых является двумерный тор $\mathbb{T}^{2}$, допускающие полиномиальные первые интегралы по скорости. Это эквивалентно полной интегрируемости системы. Полиномиальные интегралы представимы в виде многочленов по скоростям с гладкими и однозначными коэффициентами на конфигурационном пространстве. Полиномиальный интеграл $F$ наименьшей степени, независимый от интеграла энергии, назьвают неприводимым. Полиномы по скоростям, которые являются интегралами лишь для некоторых фиксированных значений полной энергии, назьвают условными полиномиальными интегралами.

В работе [1] Биркгоф исследовал локально наличие условных интегралов, являющихся полиномами не выше второй степени по скоростям. Оказалось, что наличие условного линейного интеграла связано со “скрытыми” циклическими координатами, а наличие условного квадратичного интеграла позволяет разделить канонические переменные. В п. 3 и п. 4 данной работы приведены глобальные варианты этих утверждений для случая, ког да конфигурационным пространством системы является двумерный тор.

В п. 5 уточнен результат работы [2] о полиномиальных интегралах степени $\geqslant 2$ геодезических потоков на двумерном торе.

Задача о полиномиальных интегралах степени $\leqslant 2$ рассматривалась в работах [3], [4]. В [5] показано, что натуральная механическая система на двумерном торе с кинетической энергией

$$
T=\frac{1}{2}\left(\dot{x}_{1}^{2}+\dot{x}_{2}^{2}\right)
$$

не может иметь неприводимых интегралов третьей и четвертой степени: в этом случае обязательно имеются интегралы первой и второй степени соответственно.

Работа выполнена при финансовой поддержке Российского фонда фундаментальных исследований, грант № 96-01-00747. 
В [6] рассматривалась задача о наличии полного набора независимых полиномиальных интегралов системы с торическим пространством положений $\mathbb{T}^{n}=\left\{x_{1}, \ldots, x_{n}\right.$ $\bmod 2 \pi\}$, кинетической энергией

$$
T=\frac{1}{2} \sum_{i, j} a_{i j} \dot{x}_{i} \dot{x}_{j}, \quad a_{i j}=\text { const }
$$

и потенциалом $V: \mathbb{T}^{n} \rightarrow \mathbb{R}$ в виде тригонометрического полинома. Показано, что тогда обязательно существуют $n$ независимых интегралов, степени которых $\leqslant 2$.

2. Метрика. В работе [2] рассматривался двумерньй тор с римановой метрикой

$$
d s^{2}=\frac{\left(d x_{1}^{2}+d x_{2}^{2}\right)}{2 \Lambda},
$$

где $\Lambda$ - положительная функция от $x_{1}, x_{2} \bmod 2 \pi$.

Заметим, что риманову метрику на торе не всегда можно привести к виду (1). В работе [4] отмечено, что на торе глобальные конформные координаты могут не существовать. Однако, как указано в [7], в подходящих угловых переменных $x_{1}, x_{2} \bmod 2 \pi$ на $\mathbb{T}^{2}$ метрику можно привести к следующей форме:

$$
d s^{2}=\frac{M}{2}\left(a d x_{1}^{2}+2 b d x_{1} d x_{2}+c d x_{2}^{2}\right),
$$

где функция $M: \mathbb{T}^{2} \rightarrow \mathbb{R}$ положительна, $a, b, c$ - вещественные постоянные такие, что $a c-b^{2}>0$. Для того чтобы получить метрику (2), надо воспользоваться теоремой об униформизации [8], согласно которой компактная риманова поверхность рода один конформно эквивалентна фактор-пространству комплексной плоскости $\mathbb{C}$ по некоторой решетке $P$. Если $a=c$ и $b=0$, то решетка $P$ будет ортогональной и $x_{1}, x_{2}$ - обычные конформные координаты на $\mathbb{T}^{2}$.

3. Условные линейные интегралы. Рассмотрим необратимую динамическую систему с двумя степенями свободы, конфигурационным пространством которой является двумерный тор $\mathbb{T}^{2}=\left\{x_{1}, x_{2} \bmod 2 \pi\right\}$. Пусть функция Лагранжа имеет вид

$$
L=\frac{M}{2}\left(a \dot{x}_{1}^{2}+2 b \dot{x}_{1} \dot{x}_{2}+c \dot{x}_{2}^{2}\right)+\alpha \dot{x}_{1}+\beta \dot{x}_{2}-V .
$$

Здесь $M>0, \alpha, \beta, V$ - функции от угловых координат $x_{1}, x_{2} ; a, b$ и $c$ - вещественные постоянные. Уравнения Лагранжа с лагранжианом (3) имеют интеграл энергии

$$
M\left(a \dot{x}_{1}^{2}+2 b \dot{x}_{1} \dot{x}_{2}+c \dot{x}_{2}^{2}\right)+2 V=2 h .
$$

Следуя Биркгофу [1, гл. II], выполним замену времени $t \mapsto \tau$ по формуле $d \tau=M^{-1} d t$. Обозначим штрихом дифференцирование по $\tau$.

Предположим, что нас интересуют лишь движения $x_{1}(t), x_{2}(t)$ с фиксированньгм запасом энергии $h$. Для простоты записи положим $h=0$ (в противном случае можно заменить $V$ на $V-h$ ). Траектории таких движений, параметризованные новым временем $\tau$, найдутся из системы уравнений Биркгофа:

$$
\begin{gathered}
x_{1}^{\prime \prime}=-\frac{\lambda}{\Delta}\left(b x_{1}^{\prime}+c x_{2}^{\prime}\right)-\frac{1}{\Delta}\left(c \frac{\partial \gamma}{\partial x_{1}}-b \frac{\partial \gamma}{\partial x_{2}}\right), \\
x_{2}^{\prime \prime}=\frac{\lambda}{\Delta}\left(a x_{1}^{\prime}+b x_{2}^{\prime}\right)-\frac{1}{\Delta}\left(-b \frac{\partial \gamma}{\partial x_{1}}+a \frac{\partial \gamma}{\partial x_{2}}\right), \\
\lambda=\frac{\partial \alpha}{\partial x_{2}}-\frac{\partial \beta}{\partial x_{1}}, \quad \gamma=M V, \quad \Delta=a c-b^{2}>0,
\end{gathered}
$$


дополненных энергетическим соотношением

$$
a x_{1}^{\prime 2}+2 b x_{1}^{\prime} x_{2}^{\prime}+c x_{2}^{\prime 2}+2 \gamma=0 \text {. }
$$

Используем (4) и (5) для решения задачи о наличии условных интегралов в виде полиномов не выше второй степени по скоростям. Эта задача была рассмотрена В. В. Козловым $[9$, гл. VIII $]$ для случая $a=c=1, b=0$. Распространим этот результат на общий случай.

ПРЕДЛОЖЕНИЕ 1. Предположим, что система имеет условный линейный интеграл на әнергетической поверхности $H=h$, где $h>\max V$. Тогда на пространстве положсений можно так выбрать угловые координаты $\varphi_{1}, \varphi_{2} \bmod 2 \pi u$ сделать замену времени $d t=\xi\left(\varphi_{1}, \varphi_{2}\right) d \tau$, чтобы траектории с запасом полной әнергии $h$ описывались лагранжевой системой, у которой

а) кинетическая энергия - квадратичная форма по $\varphi_{1}^{\prime}$ и $\varphi_{2}^{\prime}$ с постоянными коэффиииентами $a, b$ и с;

б) 2-форма гироскопических сил имеет вид $\lambda\left(\varphi_{1}\right) d \varphi_{1} \wedge \varphi_{2}$;

в) потенциал не зависит от переменной $\varphi_{2}$.

ДокАЗАТЕЛЬСТво. Пусть

$$
l x_{1}^{\prime}+m x_{2}^{\prime}+n
$$

- условньй линейньй интеграл. Дифференциалы $d l, d m$ и $d n$ однозначны на $\mathbb{T}^{2}=\left\{x_{1}, x_{2}\right.$ $\bmod 2 \pi\}$. Вычислим в силу системы (4) производную интеграла (6):

$$
\begin{gathered}
\frac{\partial l}{\partial x_{1}} x_{1}^{\prime 2}+\left(\frac{\partial l}{\partial x_{2}}+\frac{\partial m}{\partial x_{1}}\right) x_{1}^{\prime} x_{2}^{\prime}+\frac{\partial m}{\partial x_{2}} x_{2}^{\prime 2}+\left(\frac{1}{\Delta}(a m-b l) \lambda+\frac{\partial n}{\partial x_{1}}\right) x_{1}^{\prime} \\
+\left(\frac{1}{\Delta}(b m-c l) \lambda+\frac{\partial n}{\partial x_{2}}\right) x_{2}^{\prime}+\frac{1}{\Delta}(b m-c l) \frac{\partial \gamma}{\partial x_{1}}-\frac{1}{\Delta}(a m-b l) \frac{\partial \gamma}{\partial x_{2}}
\end{gathered}
$$

Функция (6) - интеграл уравнений (4) на энергетической поверхности (5), поэтому старшая форма многочлена (7) должна делится на энергию системы. Отсюда получаем, что

$$
c \frac{\partial l}{\partial x_{1}}-a \frac{\partial m}{\partial x_{2}}=0, \quad 2 b \frac{\partial l}{\partial x_{1}}-a\left(\frac{\partial l}{\partial x_{2}}+\frac{\partial m}{\partial x_{1}}\right)=0 .
$$

Продифференцируем первое уравнение (8) по $x_{1}$, а второе по $x_{2}$, получим уравнение на функцию $l$ :

$$
c \frac{\partial^{2} l}{\partial x_{1}^{2}}-2 b \frac{\partial^{2} l}{\partial x_{1} \partial x_{2}}+a \frac{\partial^{2} l}{\partial x_{2}^{2}}=0 .
$$

Представим функцию $l$ в виде ряда $Ф$ урье

$$
\sum_{u, v}[l]_{u v} \exp \left(i\left(u x_{1}+v x_{2}\right)\right)
$$

где $[l]_{u v}$ - коэффициенты Фурье. Решая (9) методом Фурье, получим, что точки $(u, v)$ целочисленной решетки удовлетворяют алгебраическому уравнению $c u^{2}-2 b u v+a v^{2}=0$. Поскольку метрика положительно определена, то $u=v=0$. Таким образом, $l \equiv$ const; $l=l_{0}$. Аналогично доказывается, что $m \equiv m_{0}=$ const. 
Приравнивая нулю коэффициенты при $x_{1}^{\prime}$ и $x_{2}^{\prime}$ в формуле $(7)$, получим

$$
\frac{\partial n}{\partial x_{1}}=-\frac{1}{\Delta}(a m-b l) \lambda, \quad \frac{\partial n}{\partial x_{2}}=-\frac{1}{\Delta}(b m-c l) \lambda .
$$

Форма $d n$ однозначна, поэтому

$$
\left(c l_{0}-b m_{0}\right) \frac{\partial \lambda}{\partial x_{1}}+\left(-b l_{0}+a m_{0}\right) \frac{\partial \lambda}{\partial x_{2}}=0 .
$$

Приравнивая нулю (7), получим аналогичное соотношение для потенциала:

$$
\left(c l_{0}-b m_{0}\right) \frac{\partial \gamma}{\partial x_{1}}+\left(-b l_{0}+a m_{0}\right) \frac{\partial \gamma}{\partial x_{2}}=0
$$

Если выражения $c l_{0}-b m_{0}$ и $-b l_{0}+a m_{0}$ рационально несоизмеримы (типичная ситуация), то из (10) и (11) вытекает постоянство функций $\lambda, \gamma$ и тогда предложение 1 доказано. Пусть

$$
\frac{c l_{0}-b m_{0}}{-b l_{0}+a m_{0}}=\frac{p}{q}
$$

причем целые числа $p$ и $q$ взаимно просты. Из (10) и (11) легко вьводится, что $\lambda=\chi \times$ $\left(q x_{1}-p x_{2}\right), \gamma=\psi\left(q x_{1}-p x_{2}\right)$, где функции $\chi$ и $\psi 2 \pi$-периодичны. Выполним линейное преобразование $\varphi_{1}=q x_{1}-p x_{2}, \varphi_{2}=r x_{1}+s x_{2}$ с целыми $r, s$, удовлетворяющими соотношению $q s+p r=1$. По теореме Безу ввиду взаимной простоты $p$ и $q$ такие числа $r$ и $s$ сушествуют. Переменные $\varphi_{1}, \varphi_{2} \bmod 2 \pi$ - искомые.

4. Условные квадратичные интегралы. Теперь рассмотрим обратимьй случай, когда $\alpha=\beta=0$. Разложим потенциал $\gamma$ в ряд Фурье

$$
\sum_{u, v}[\gamma]_{u v} \exp \left(i\left(u x_{1}+v x_{2}\right)\right)
$$

Cпектром функции $\gamma$ назовем подмножество целочисленной решетки:

$$
S_{\gamma}=\left\{w=(u, v) \in \mathbb{Z}^{2}:[\gamma]_{u v} \neq 0\right\}
$$

Оно переходит в себя при отражении $w \mapsto-w$.

ПРЕДЛОЖЕНИЕ 2. Пусть $h>\max V$ и система имеет условный квадратичный интеграл с однозначными коэффичиентами. Тогда на пространстве положений можсно так выбрать угловые координаты $\varphi_{1}, \varphi_{2} \bmod 2 \pi$ и сделать замену времени $d t=\xi\left(\varphi_{1}, \varphi_{2}\right) d \tau$, чтобы траектории движений с запасом полной энергии $h$ описывались лагранжевой системой, у которой

а) кинетическая әнергия - квадратичная форма по $\varphi_{1}^{\prime}$ и $\varphi_{2}^{\prime}$ с постоянными коэффиииентами $a, b$ и

б) спектр $S_{\gamma}$ лежит на двух прямых, ортогонально пересекающихся в начале координат в метрике, обратной исходной. 
ЗАмЕчАниЕ. В типичном случае спектр $S_{\gamma}$ лежит на одной прямой и тогда условньй квадратичньй интеграл сводится к линейному условному интегралу.

Для доказательства предложения снова воспользуемся уравнениями (4), полагая $\lambda \equiv 0$. Предположим, что уравнения (4) имеют квадратичньй интеграл

$$
\frac{1}{2}\left(A x_{1}^{\prime 2}+2 B x_{1}^{\prime} x_{2}^{\prime}+C x_{2}^{\prime 2}\right)+f
$$

на поверхности (5). Выпишем члены третьей степени по скоростям в выражении для производной интеграла (12) по новому времени $\tau$ :

$$
\frac{1}{2} \frac{\partial A}{\partial x_{1}} x_{1}^{\prime 3}+\left(\frac{\partial B}{\partial x_{1}}+\frac{1}{2} \frac{\partial A}{\partial x_{2}}\right) x_{1}^{\prime 2} x_{2}^{\prime}+\left(\frac{\partial B}{\partial x_{2}}+\frac{1}{2} \frac{\partial C}{\partial x_{1}}\right) x_{1}^{\prime} x_{2}^{\prime 2}+\frac{1}{2} \frac{\partial C}{\partial x_{2}} x_{2}^{\prime 3} .
$$

Так как (12) - условньй интеграл, то этот полином (13) должен делиться на полную энергию, т.е. его можно представить в виде:

$$
\left(K x_{1}^{\prime}+L x_{2}^{\prime}\right)\left(a x_{1}^{\prime 2}+2 b x_{1}^{\prime} x_{2}^{\prime}+c x_{2}^{\prime 2}\right) .
$$

Приравняем коэффициенты при одинаковых степенях $x_{1}^{\prime}$ и $x_{2}^{\prime}$ в (13) и (14):

$$
\begin{aligned}
K a & =\frac{1}{2} \frac{\partial A}{\partial x_{1}}, & 2 K b+L a=\frac{\partial B}{\partial x_{1}}+\frac{1}{2} \frac{\partial A}{\partial x_{2}} \\
L c & =\frac{1}{2} \frac{\partial C}{\partial x_{2}}, & 2 L b+K c=\frac{\partial B}{\partial x_{2}}+\frac{1}{2} \frac{\partial C}{\partial x_{1}} .
\end{aligned}
$$

Отсюда получаем два уравнения:

$$
a c\left(\frac{\partial B}{\partial x_{1}}+\frac{1}{2} \frac{\partial A}{\partial x_{2}}\right)=b c \frac{\partial A}{\partial x_{1}}+\frac{1}{2} a^{2} \frac{\partial C}{\partial x_{2}}, \quad a c\left(\frac{\partial B}{\partial x_{2}}+\frac{1}{2} \frac{\partial C}{\partial x_{1}}\right)=\frac{1}{2} c^{2} \frac{\partial A}{\partial x_{1}}+b a \frac{\partial C}{\partial x_{2}} .
$$

Эти уравнения можно представить в следующей форме:

$$
2 c \frac{\partial(a B-b A)}{\partial x_{1}}-a \frac{\partial(a C-c A)}{\partial x_{2}}=0, \quad 2 a \frac{\partial(c B-b C)}{\partial x_{2}}+c \frac{\partial(a C-c A)}{\partial x_{1}}=0 .
$$

Продифференцируем второе уравнение (15) по $x_{1}$, а первое по $x_{2}$ и выгтем из второго уравнения первое; получим уравнение, аналогичное (9):

$$
c \frac{\partial^{2}}{\partial x_{1}^{2}}(a C-c A)-2 b \frac{\partial^{2}}{\partial x_{1} \partial x_{2}}(a C-c A)+a \frac{\partial^{2}}{\partial x_{2}^{2}}(a C-c A)=0 .
$$

Для решения этого уравнения также воспользуемся методом Фурье. Тогда

$$
a C-c A=k_{2}, \quad k_{2}=\text { const }
$$

Используя (16), из уравнений (15) получим еще два равенства

$$
c B-b C=k_{1}, \quad b A-a B=k_{3},
$$

где $k_{2}, k_{3}$ - вещественные постоянные. Заметим, что из равенств (16) и (17) вытекает соотношение

$$
a k_{1}+b k_{2}+c k_{3}=0
$$


Используя интеграл энергии (5), квадратичньй интеграл (12) можно преобразовать к виду, в котором коэффициенты $A, B$ и $C$ будут удовлетворять равенствам (16) и (17). Дифференцируя еще раз интеграл (12) в силу системы (4) и приравнивая нулю коэффициенты при $x_{1}^{\prime}$ и $x_{2}^{\prime}$, получим соотношения:

$$
\begin{aligned}
& \frac{A}{\Delta}\left(c \frac{\partial \gamma}{\partial x_{1}}-b \frac{\partial \gamma}{\partial x_{2}}\right)+\frac{B}{\Delta}\left(-b \frac{\partial \gamma}{\partial x_{1}}+a \frac{\partial \gamma}{\partial x_{2}}\right)=\frac{\partial f}{\partial x_{1}} \\
& \frac{B}{\Delta}\left(c \frac{\partial \gamma}{\partial x_{1}}-b \frac{\partial \gamma}{\partial x_{2}}\right)+\frac{C}{\Delta}\left(-b \frac{\partial \gamma}{\partial x_{1}}+a \frac{\partial \gamma}{\partial x_{2}}\right)=\frac{\partial f}{\partial x_{2}}
\end{aligned}
$$

Следовательно, для функции $\gamma$ получим следующее уравнение:

$$
(c B-b C) \frac{\partial^{2} \gamma}{\partial x_{1}^{2}}+(a C-c A) \frac{\partial^{2} \gamma}{\partial x_{1} \partial x_{2}}+(b A-a B) \frac{\partial^{2} \gamma}{\partial x_{2}^{2}}=0
$$

или

$$
k_{3} \frac{\partial^{2} \gamma}{\partial x_{2}^{2}}+k_{2} \frac{\partial^{2} \gamma}{\partial x_{1} \partial x_{2}}+k_{1} \frac{\partial^{2} \gamma}{\partial x_{1}^{2}}=0
$$

Применяя метод Фурье, получим

$$
k_{3} v^{2}+k_{2} v u+k_{1} u^{2}=0
$$

для всех $(u, v) \in S_{\gamma}$. Введем новую переменную $\operatorname{tg} \vartheta=v / u$, где $\vartheta$ - угол между лучом, проходящим через вершину $(u, v) \in S_{\gamma}$, и горизонтальной координатной осью. Тогда

$$
k_{3} \operatorname{tg}^{2} \vartheta+k_{2} \operatorname{tg} \vartheta+k_{1}=0 .
$$

В случае $k_{3}=0$ (т.е. $b / a=B / A$ согласно (17)) уравнение (19) становится линейным. С учетом (18) получаем

$$
\operatorname{tg} \vartheta=-\frac{k_{1}}{k_{2}}=\frac{b}{a}
$$

В этом случае спектр $S_{\gamma}$ лежит на одной прямой, проходящей через начало координат. Подсчитаем дискриминант квадратного уравнения (19):

$$
D=\left(k_{2}+\frac{2 b}{a} k_{3}\right)^{2}+\frac{4\left(a c-b^{2}\right)}{a^{2}} k_{3}^{2} .
$$

Если $D=0$ (т.е. $\left.k_{3}=k_{2}=0\right)$, то из (18) или (19) следует равенство $k_{1}=0$. Тогда из (16) и (17) имеем

$$
\frac{a}{c}=\frac{A}{C}, \quad \frac{b}{a}=\frac{B}{A}, \quad \frac{b}{c}=\frac{B}{C},
$$

т.е. интеграл (12) пропорционален интегралу энергии (5), что противоречит его неприводимости.

Рассмотрим теперь случай, когда $D>0$, тогда у квадратного уравнения (19) есть два различных корня $\operatorname{tg} \vartheta_{1}$ и $\operatorname{tg} \vartheta_{2}$. Пусть

$$
\operatorname{tg} \vartheta_{1}=\frac{v_{1}}{u_{1}}, \quad \operatorname{tg} \vartheta_{2}=\frac{v_{2}}{u_{2}} .
$$


Это - случай, когда спектр $S_{\gamma}$ лежит на двух прямых. Рассмотрим условие ортогонального пересечения полученных прямых в обратной метрике:

$$
\left(\begin{array}{ll}
u_{2} & v_{2}
\end{array}\right)\left(\begin{array}{cc}
c & -b \\
-b & a
\end{array}\right)\left(\begin{array}{l}
u_{1} \\
v_{1}
\end{array}\right)=0
$$

или

$$
c u_{2} u_{1}-b u_{2} v_{1}+a v_{2} v_{1}-b u_{1} v_{2}=0 .
$$

Разделим (21) на $u_{1} u_{2}$ и, учитьвая $(20)$, получим

$$
a \operatorname{tg} \vartheta_{1} \operatorname{tg} \vartheta_{2}-b\left(\operatorname{tg} \vartheta_{1}+\operatorname{tg} \vartheta_{2}\right)+c=0
$$

По теореме Виета

$$
a \frac{k_{1}}{k_{3}}+b \frac{k_{2}}{k_{3}}+c=0,
$$

что эквивалентно соотношению (18).

Заметим, что если вьполняются отношения $k_{2} / k_{3} \in \mathbb{Q}$ и $k_{1} / k_{3} \in \mathbb{Q}$, то обе прямые проходят через начало координат и целочисленные точки на плоскости, откуда следует наличие неприводимого квадратичного интеграла.

5. Полиномиальные интегралы степени выше 2. В работе [2] рассматривалась задача о полиномиальных интегралах геодезических потоков на двумерном торе для метрики (2), у которой $a=c$ и $b=0$. Установлено, что если $M-$ тригонометрический многочлен (спектр $M$ конечен) и если геодезический поток допускает дополнительный неприводимьй интеграл, полиномиальный по импульсам, то его степень не превосходит 2. Оказывается, этот результат можно распространить на случай произвольных метрик (2), если снова предположить, что конформньй множитель $M$ является тригонометрическим многочленом.

ТЕОремА 1. Предположим, что имеется полиномиальный по скоростям интеграл $F$, независимый от интеграла энергии. Тогда

1) если степень $F$ нечетна, то спектр функиии $M$ лежст на одной прямой, проходящей через начало координат;

2) если степень $F$ четна, то спектр функиии $M$ лежит на двух прямых, ортогонально пересекающихся в начале координат в метрике, обратной исходной.

СлЕДСТВИЕ. Если уравнения геодезических на торе с метрикой (2), әде $M$ тригонометрический многочлен, допускают дополнительный полиномиальный интеграл $F$, то найдется независимый от интеграла әнергии полиномиальный интеграл степени $\leqslant 2$. Причем, если степень $F$ нечетна, то существует линейный интеграл.

ЗАмЕчАНИЕ. В типичной ситуации нет ортогональных прямых, проходящих через начало координат и целочисленные точки на плоскости. Поэтому наличие неприводимого квадратичного интеграла может быть лишь в исключительных случаях.

Теорема 1 доказывается методом работы [2]. Для этого приведем квадратичную формy

$$
a \dot{x}_{1}^{2}+2 b \dot{x}_{1} \dot{x}_{2}+c \dot{x}_{2}^{2}
$$


к сумме квадратов $\dot{q}_{1}^{2}+\dot{q}_{2}^{2}$ с помощью линейного преобразования

$$
\left(\begin{array}{l}
x_{1} \\
x_{2}
\end{array}\right)=\Gamma\left(\begin{array}{l}
q_{1} \\
q_{2}
\end{array}\right), \quad|\Gamma| \neq 0 .
$$

В общем случае это преобразование не является автоморфизмом тора $\mathbb{T}^{2}$, и поэтому $q_{1}, q_{2}$ - конформные координаты на накрываюшей тор плоскости. В этих переменных метрика (2) имеет конформньй вид

$$
d s^{2}=\frac{\widetilde{M}}{2}\left(d q_{1}^{2}+d q_{2}^{2}\right) .
$$

Если

$$
M=\sum_{w=(u, v)}[M]_{w} \exp (i(w, x)), \quad w=(u, v),
$$

- тригонометрический многочлен, то

$$
\widetilde{M}=\sum_{w=(u, v)}[M]_{w} \exp \left(i\left(\Gamma^{T} w, q\right)\right)
$$

будет конечной тригонометрической суммой, уже не периодической по переменным $q_{1}, q_{2}$. Суммы вида (23) играют роль рядов Фурье и с ними можно оперировать по тем же правилам, что и с обычными рядами Фурье.

Используя метод [2], на целочисленные векторы $w \neq 0$ из (23) получаются те же геометрические соотношения, но только обычная евклидова метрика заменена на метрику (22). Анализ этих соотношений несколько усложняется (например, в отличие от [2] для анализа интеграла нечетной степени приходится вводить присоединенные вершины для спектра функции $\widetilde{M})$. Однако, в итоге снова получаем, что спектр функции $M$ лежит на одной или двух прямых, ортогональных в метрике, двойственной (2).

Автор выражает благодарность В.В. Козлову за внимание и помощь в работе.

\section{СПИСОК ЦИТИРОВАННОЙ ЛИТЕРАТУРЫ}

[1] Биркгоф Дж. Д. Динамические системы. М.-Л.: Гостехиздат, 1941.

[2] Козлов В.В., Денисова Н. В. Полиномиальные интегралы геодезических потоков на двумерном торе // Матем. сб. 1994. Т. 185. №12. С. 49-64.

[3] Колокольцов В. Н. Геодезические потоки на двумерных многообразиях с дополнительным полиномиальным по скоростям первым интегралом // Изв. АН СССР. Сер. матем. 1982. Т. 46. № 5. C. 994-1010.

[4] Бабенко И. К., Нехорошев Н. Н. О комплексных структурах на двумерных торах, допускающих метрики с нетривиальным квадратичным интегралом // Матем. заметки. 1995. T. 58. № 5. C. $643-652$.

[5] Бялый М. Л. О полиномиальных по импульсам первых интегралах для механической системы на двумерном торе // Функцион. анализ и его прилож. 1987. Т. 21. № 4. С. 64-65.

[6] Козлов В.В., Трещёв Д.В. Об интегрируемости гамильтоновых систем с торическим пространством положений // Матем. сб. 1988. Т. 135 (177). №1. С. 119-138.

[7] Козлов В.В. О периодических траекториях заряда в магнитном поле // Регулярная и хаотическая динамика. (в печати).

[8] Гурвиц А., Курант Р. Теория функций. М.: Наука, 1968.

[9] Козлов В. В. Симметрии, топология и резонансы в гамильтоновой механике. Ижевск: УдГУ, 1995. 